\title{
EFFECT OF VARIOUS PH MEDIA ON SOME OPTICAL PROPERTIES AND SURFACE ROUGHNESS OF BILAYERED REINFORCED POLY- MER AND MONOLITHIC HYBRID CERAMIC (AN IN-VITRO STUDY)
}

\author{
Ramy F. Hussein*, Hussein R. Mohammed**, Khaled M. Haggag***
}

\begin{abstract}
Objective: The objective of this study was to evaluate the effect of different $\mathrm{pH}$ media on color stability, translucency and surface roughness on Hybrid ceramic material (Vita Enamic) and bilayered reinforced polymer (PEEK). Materials and methods: A total of 60 discs, 30 in each group that were designed with milled with CAD/CAM, each group was further subdivided into 3 subgroups (10 samples in each group) according to the immersing solution.(neutral, weak acid and strong acid), all samples were aged in the immersion solutions for 24 days in an incubator at $37^{\circ}$ which simulate 2 years of clinical service. Optical profilometer was used to assess surface roughness, a spectrophotometer was used to assess color change and translucency was calculated with the tranclucency parameter equation. Results: The results showed that no statistical significant difference in surface roughness, color stability and translucency with Vita Enamic in different solutions, and statistical significant difference with PEEK group in different solutions. Conclusion: Vita Enamic has higher color stability and translucency than PEEK and can maintain smoother surface with different $\mathrm{pH}$ media.
\end{abstract}

KEYWORDS: ph Media, Optical Properties, Surface Roughness, PEEK, Vita Enamic

\section{INTRODUCTION}

With the increased demand in esthetics, patients and dentists are seeking perfection in restoring natural teeth. Ceramics have shown the most accurate reproduction of the appearance, color, and texture of natural teeth ${ }^{(1)}$. Several ceramicbased materials have recently been introduced for CAD-CAM dentistry including lithium disilicate glass ceramic, yttria-stabilized tetragonal zirconia polycrystalline (Y-TZP), monolithic zirconia, and zirconia-containing lithium silicate ceramics nanoresin ceramic, hybrid ceramic, interpenetrating phase ceramic. These materials help in the technological revolution that offers clinicians appropriate ceramics with the ease of restoration fabrication through CAD-CAM technology ${ }^{(2)}$. Vita Enamic is a member of hybrid ceramics group by VITA;
The ceramic part consists of an aluminum oxideenriched, fine-structure feldspar matrix (86 wt.\%) infused by a polymer material consisting of (14 wt $\%$ ) urethane dimethacrylate and triethylene glycol dimethacrylate. Its flexural strength is $151 \mathrm{MPa}$. (3) Both advantages of ceramic and resin materials are combined in ceramic/polymer materials such as less brittleness, excellent machinability and edge stability ${ }^{(3)}$.

New materials based on polyether ether ketone (PEEK) were introduced to the market as a potential alternative material for surgical procedures such as inter-body fusion cages or dental implants with a similar stress distribution as titanium implants ${ }^{(4)}$. PEEK is an organic thermoplastic material with excellent mechanical and chemical resistance properties. Another variant of PEEK is BioHPP

\footnotetext{
* Demonstrator of Crown \& Bridge department, Faculty of Dental Medicine, MTI University.

** Lecturer of Crown \& Bridge department, Faculty of Dental Medicine (Cairo, Boys), Al Azhar University.

*** Professor of Crown \& Bridge department, Faculty of Dental Medicine (Cairo, Boys), Al Azhar University.
} 
developed by Bredent GmbH. BioHPP stands for Bio High Performance Polymer and has been developed especially for dental applications. The material has been proven to be very popular amongst dental technicians ${ }^{(5)}$.

One of the problems that face hybrid ceramics is long term color stability, discoloration may be endogenous or exogenous. Endogenous discoloration results from chemical instability of the material, exogenous staining results from adsorption of stains from oral cavity and is affected by other conditions as surface roughness. ${ }^{(6)}$ With the increasing demand on aesthetic restorations, it is necessary to evaluate the color stability of these hybrid materials when it is subjected to different $\mathrm{pH}$ media. Commonly consumed beverages can range from neutral solutions, weak acids and strong acids ${ }^{(7)}$.

The aim of this study was to evaluate the effect of different $\mathrm{pH}$ media on color stability, translucency and surface roughness on Hybrid ceramic material (Vita Enamic) and bilayered reinforced polymer (PEEK).

\section{MATERIALS AND METHODS}

\section{Samples grouping:}

A total of 60 disc shaped samples with dimensions (10mm diameter x $1.5 \mathrm{~mm}$ thickness) were fabricated. They were divided into two equal groups of 30 samples each according to the type of material used: (Group I PEEK and Group II Vita Enamic). PEEK samples were composed of two layers; (substrate layer Bio HPP and the veneering layer HIPC).

\section{Samples fabrication}

One blank of each Bio.HPP and Bio.HIPC were used to obtain cylinders with diameter of $(10 \mathrm{~mm})$, $\mathrm{CAD} / \mathrm{CAM}$ was used to design and mill cylinder shapes, these cylinders were cut into 30 discs of Bio. HPP and 30 discs of HIPC with desired dimensions (10mm diameter $\mathrm{x} 0.7 \mathrm{~mm}$ thickness) and 30 discs of Bio.HIPC (10mm diameter x $0.8 \mathrm{~mm}$ thickness) using Isomet cutting machine (Isomet 4000 linear precision saw, Lake Bluff, USA) (2500rpm with coolant), digital caliper (Pro'skit Mechanical digital caliper) was used to insure thickness and diameter of each sample. Cementation of HIPC discs on Bio. Hpp discs was done according to manufacturer instructions by the following steps.

First the veneering surface of Bio.Hpp and the luting surface of HIPC discs were abraded with air-

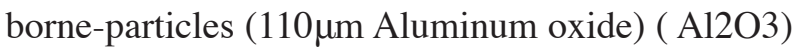
powder (basic Quattro IS; Renfert, Hilzingen, Germany) with pressure $(2 \mathrm{MPa})$, at an angle of $45^{\circ}$ from a distance of $10 \mathrm{~mm}$ using customized sandblasting machine, and subsequently put in an ultrasonic bath (L\&R Transistor Ultrasonic T14, L\&R, Kearny, NY, USA) filled with distilled water for 5 min, Afterwards, then handled with plastic tweezers and left to dry.

Bio.Hpp and HIPC discs were conditioned using visio.link for 1 minute and polymerized for 90 seconds at $220 \mathrm{~mW} / \mathrm{cm}^{2} 90 \mathrm{~s}$ by polymerizing light cure machine (Bre.Lux power unit 2-bredent $\mathrm{GmbH} \& \mathrm{Co} . \mathrm{KG}){ }^{(8)}$ A thin layer of combo.lign luting cement was applied to bonding surfaces of HIPC before pressing them on the Bio.Hpp discs, then excess cement was removed by brush and then samples were polymerized by light cure machine for $180 \mathrm{~s}$ at $220 \mathrm{~mW} / \mathrm{cm}^{2}{ }^{(8)}$.

The Vita Enamic block were designed and milled by the same technique used with PEEK to obtain cylinder blocks with round diameter then these cylinders were cut by micro saw to obtain 30 round shaped samples with surface area of $10 \mathrm{~mm}$ diameter and $1.5 \mathrm{~mm}$ thickness.

An experienced technician carried out the finishing and polishing sequences in accordance with the manufacturer's instructions for rpm. The same straight hand piece and electric motor was used to control the rpm setting for each step. 


\section{Aging:}

Base line readings (surface roughness, color and translucency) were measured for each sample before aging process, then each sample was stored in a separate container, These containers were filled with different $\mathrm{pH}$ solutions according to each group.

The total storage time was 24 days in an incubator at $37^{\circ} \mathrm{C}$ which simulate 2 years of clinical service ${ }^{(9)}$. The solutions were changed every 24 hours and fresh solution was prepared each time.

\section{Surface roughness measurements:}

The optical methods tend to fulfill the need for quantitative characterization of surface topography without contact ${ }^{(10)}$. Samples were photographed using optical profilometer, USB Digital microscope with a built-in camera (U500X Capture Digital Microscope, Guangdong, China) connected with an IBM compatible personal computer using a fixed magnification of $120 \mathrm{X}$. The images were recorded with a resolution of $1280 \times 1024$ pixels per image. Digital microscope images were cropped to 350 x 400 pixels using Microsoft office picture manager to specify/standardize area of roughness measurement. The cropped images were analyzed using WSxM software (Ver 5 develop 4.1, Nanotec, Electronica, SL) ${ }^{(11)}$. Within the WSxM software, all limits, sizes, frames and measured parameters are expressed in pixels. Therefore, system calibration was done to convert the pixels into absolute real world units. Calibration was made by comparing an object of known size (a ruler in this study) with a scale generated by the software. Subsequently, a 3D image of the surface profile of the samples was created. Five 3D images were collected for each sample, in the central area and in the sides at area of $10 \mu \mathrm{m} \times 10 \mu \mathrm{m}^{(12)}$.

WSxM software was used to calculate average of heights ( $\mathrm{Ra}$ ) expressed in $\mu \mathrm{m}$, which can be assumed as a reliable indices of surface roughness ${ }^{(13)}$.

\section{Color change $(\Delta \mathrm{E})$ measurements:}

Color stability was assessed using spectrophotometer each sample was assessed before and after aging, The color was measured using a reflective spectrophotometer (X-Rite, model RM200QC, Neu-Isenburg, Germany). The aperture size was set to $4 \mathrm{~mm}$ and the specimens were exactly aligned with the device. A white background was selected and measurements were made according to the CIE L*a*b* color space relative to the CIE standard illuminant D65. The color changes $(\Delta \mathrm{E})$ of the specimens were evaluated using the following formula: $\Delta \mathrm{E}_{\text {CIELAB }}=\left(\Delta \mathrm{L}^{* 2}+\Delta \mathrm{a}^{* 2}+\Delta \mathrm{b}^{* 2}\right) \frac{1 / 2}{2}$

\section{Translucency parameters (TP)}

The translucency parameters (TP) values were obtained by calculating the color difference of the samples over black and white backgrounds by using the following equation: $\mathrm{TP}=\left[\left(\mathrm{L}_{\mathrm{b}}^{*}-\mathrm{L}_{\mathrm{w}}{ }^{*}\right)^{2}+\left(\mathrm{a}_{\mathrm{b}}^{*}-\right.\right.$ $\left.\left.\mathrm{a}_{\mathrm{w}}{ }^{*}\right)^{2}+\left(\mathrm{b}_{\mathrm{b}}{ }^{*}-\mathrm{b}_{\mathrm{w}}{ }^{*}\right)^{2}\right]^{1 / 2}$, where letters "b" and "w" refer to color coordinates over the black and white backgrounds, respectively.

\section{RESULTS AND STATISTICAL TESTS}

The results were analyzed using Graph Pad Instat (Graph Pad, Inc.). A value of $\mathrm{P}<0.05$ was considered statistically significant, one-way ANOVA was done for compared time followed by Tukey's pair-wise if showed significant. Two-way analysis of variance was performed. Student t-test was done between groups at different $\mathrm{pH}$ media.

\section{Roughness}

For $\boldsymbol{G r} \_\boldsymbol{P E E K}$; it was found that a statistically significant in roughness mean value between subgroups. For $\boldsymbol{G r}$-Vita Enamic: it was found that no statistical significant difference in roughness mean value between subgroups. All values are represented in table 1 . 
TABLE (1) Descriptive statistics of roughness results (Mean values \pm SDs) in $\mu \mathrm{m}$ for both groups at different $\mathrm{pH}$ media:

\begin{tabular}{|c|c|c|c|c|c|}
\hline Variables & Baseline & pH_5.5 & pH_2.5 & $p H_{-} 7$ & Pvalue \\
\hline $\begin{array}{c}G r_{-} \\
P E \bar{E} K\end{array}$ & $\begin{array}{c}0.2241^{\text {b в }} \\
\pm 0.014\end{array}$ & $\begin{array}{l}0.2439^{\mathrm{a}} \\
{ }^{\mathrm{B}} \pm 0.013\end{array}$ & $\begin{array}{c}0.2189^{\mathrm{b} \text { в }} \\
\pm 0.008\end{array}$ & $\begin{array}{r}0.2384^{\mathrm{a}} \\
{ }^{\mathrm{B}} \pm 0.019\end{array}$ & $0.0005^{*}$ \\
\hline $\begin{array}{l}\text { Gr_Vita } \\
\text { Enamic }\end{array}$ & $\begin{array}{c}0.2453 \\
\pm 0.009 \text { A }\end{array}$ & $\begin{array}{c}0.2541 \\
\pm 0.006^{\mathrm{A}}\end{array}$ & $\begin{array}{c}0.2492 \\
\pm 0.013 \mathrm{~A}\end{array}$ & $\begin{array}{c}0.2510 \\
\pm 0.006^{\mathrm{A}}\end{array}$ & 0.2259 \\
\hline Pvalue & $0.0013^{*}$ & $0.0306^{*}$ & $<0.0001 *$ & $0.0194^{*}$ & \\
\hline
\end{tabular}

* Indicates the mean difference is statistically significant at the 0.05 level.

Different small letters indicate statistically significant difference in the same row. (p-value $\leq 0.05)$.

Different capital letters indicate statistically significant difference in the same column. (p-value $\leq 0.05)$.

\section{Color change $(\Delta \mathrm{E})$ measurements:}

For $\boldsymbol{G r} \_\boldsymbol{P E E K}$ : it was found that a statistically significant in $\triangle \mathrm{E}$ mean value between subgroups. For $\boldsymbol{G r}$ _Vita enamic: it was found that no statistical significant difference in $\Delta \mathrm{E}$ mean value between subgroups. All values are represented in table 2.

TABLE (2): Descriptive statistics of color change results (Mean values \pm SDs) for both groups at different $\mathrm{pH}$ media

\begin{tabular}{|c|c|c|c|c|}
\hline Variables & $\boldsymbol{p H}$ _5.5 & $\boldsymbol{p H} \boldsymbol{H}_{-} .5$ & $\boldsymbol{p H}$ _7 & P value \\
\hline Gr_PEEK & $8.07 \pm 3.9 \mathrm{a}$ & $4.29 \pm 1.27^{\mathrm{b}}$ & $5.89 \pm 1.51^{\mathrm{ab}}$ & $0.0097^{*}$ \\
\hline $\begin{array}{l}\text { Gr_Vita } \\
\text { Enamic }\end{array}$ & $7.95 \pm 2.44$ & $5.46 \pm 1.34$ & $7.02 \pm 4.33$ & $0.1862 \mathrm{~ns}$ \\
\hline P value & $0.9439 \mathrm{~ns}$ & $0.059 \mathrm{~ns}$ & $0.4473 \mathrm{~ns}$ & \\
\hline
\end{tabular}

\section{Translucency parameters (TP):}

ForGr_PEEK:itwas found thatnostatisticalsignificant difference inTPmean value between subgroups. For $\boldsymbol{G r}$ _Vita Enamic: it was found that no statistical significant difference in TP mean value between subgroups.
TABLE (3): Descriptive statistics of translucency parameter results (Mean values \pm SDs) in TP for both groups at different $\mathrm{pH}$ media

\begin{tabular}{|c|c|c|c|c|c|}
\hline Variables & Baseline & pH_5.5 & $p H \_2.5$ & $p H \_7$ & Pvalue \\
\hline $\begin{array}{c}G r_{-} \\
P E E K\end{array}$ & $\begin{array}{c}7.61 \\
\pm 2.32 \text { в }\end{array}$ & $\begin{array}{c}7.76 \\
\pm 2.69^{\text {в }}\end{array}$ & $\begin{array}{c}6.99 \\
\pm 2.49^{\text {в }}\end{array}$ & $\begin{array}{c}6.97 \\
\pm 3.34^{\text {в }}\end{array}$ & $0.8245 \mathrm{~ns}$ \\
\hline $\begin{array}{l}\text { Gr_Vita } \\
\text { Enamic }\end{array}$ & $\begin{array}{c}16.33 \\
\pm 3.21^{\mathrm{A}}\end{array}$ & $\begin{array}{c}16.72 \\
\pm 4.56^{\mathrm{A}}\end{array}$ & $\begin{array}{c}14.25 \\
\pm 3.07 \mathrm{~A}\end{array}$ & $\begin{array}{l}12.82 \\
\pm 3.8^{\mathrm{A}}\end{array}$ & $0.0580 \mathrm{~ns}$ \\
\hline Pvalue & $<0.0001^{*}$ & $<0.0001^{*}$ & $<0.0001^{*}$ & $0.0018^{*}$ & \\
\hline
\end{tabular}

\section{DISCUSSION}

Color of dental restorative material is one of the optical properties, and it is the visual perception of an object, or substance regarding the light reflected, or transmitted through ${ }^{(14)}$. Tooth-colored fillings and fixed dental prostheses are important expectations to achieve patients and dentists satisfaction.

Color stability can be affected by many factors such as: incomplete polymerization, chemical reactivity, water sorption, and surface roughness of material. ${ }^{(15,16)}$ Polishing ability and material structure are not the only parameters providing color stability for a longer period of time but also patient's dietary habits and cleaning measures are key factors ${ }^{(17,18)}$. With aging process in clinical use, ion leaching out occurs leads to surface roughness of the material and subsequent color change ${ }^{(19)}$.

In this study these two different materials were selected because both of them have polymer phases. PEEK (Bio.Hpp) and veneering composite (Brecam.HIPC) were used not the granules type because it is a CAD/CAM material same as Vita Enamic.

Polyetheretherketone (PEEK) is a semicrystalline thermoplastic polymer and is considered as a promising alternative of fixed and removable Prosthetic dentistry with adequate mechanical properties to fulfill the basic requirements in the restorative field as showed by recent studies ${ }^{(20)}$. 
Vita Enamic is one of the polymer-infiltrated feldspathic ceramic materials, consists of $86 \%$ ceramic (by weight) ${ }^{(21)}$. Besides having the properties of both ceramic and composite materials, polymer-infiltrated ceramic network (PICN) materials are considered to have mechanical and aesthetic properties similar to natural teeth ${ }^{(22)}$. Highly aesthetic milling block that provides an alternative to ceramic blocks for computer-aided design/computer-aided manufacturing $(\mathrm{CAD} / \mathrm{CAM})$ indirect restorations $^{(23)}$.

This present study was performed in vitro to evaluate the effect of different $\mathrm{pH}$ media on surface roughness and color stability of different hybrid materials; In-vitro testing was used because it overcomes the limitations associated with the clinical testing such as individual variation by creating a controlled environment. These tests provide a guideline about the color stability of the two materials and act as a baseline for the clinical studies $^{(24)}$.

The supporting structure or esthetic restorative material is a primary source of the resulting color. This color is influenced by the thickness and translucency of the final restoration, as evident by the amount of reflection and scattering of light ${ }^{(25)}$.

Samples where cut into discs with the diameter $10 \mathrm{~mm}$ and $1.5 \mathrm{~mm}$ thickness, these sample configuration was selected because it fits experimental system, also it could be considered adequate thickness recommended by the manufacturer for prosthetic appliance. Peek samples were veneered to simulate clinical conditions, there are different systems and techniques for the peek and veneering fabrication.

Another criterion of aesthetics is the surface roughness. Rough surfaces are more prone to coloration because of its elevated stain retention capacity ${ }^{(26-28)}$. The evidence proved that stain resistance and color stability is improved with properly polished surfaces ${ }^{(29)}$. It was also reported that materials with different microstructures require different polishing techniques ${ }^{(30)}$, so that polishing was per- formed by the same operator for both groups but with different polishing kits. Peek samples were polished with Bredent polishing kit, and Vita Enamic samples were polished using the Vita Enamic technical polishing set following the manufacturer's instructions.

In the oral cavity restorative materials are continuously exposed to different $\mathrm{pH}$ media according to dietary habits and oral conditions. The total immersion time was 24 days is equivalent to 2 years of consumption of the beverages which might be enough time to evaluate clinical success ${ }^{(9)}$.

\section{Surface roughness}

Samples were photographed using USB Digital microscope because of easier access, affordability and reduced time.

It was found that in baseline readings Vita Enamic has higher statistical significant surface roughness than PEEK group, but within the subgroups there was significant change with different $\mathrm{pH}$ media on PEEK and no significant change on Vita Enamic group.

Between all pH media, Pepsi subgroup showed lowest statistically significant decrease in surface roughness $(0.2189 \pm 0.008 \mu \mathrm{m})$, the low $\mathrm{pH}$ of Pepsi( 2.5) may have affected the surface integrity of PEEK veneering resulting in smoothening of the surface $^{(31)}$.

Cruz et al. ${ }^{(32)}$ stated that the increase in surface roughness of Vita Enamic with exposure to strong acids was possibly due to the dissolution of the ceramic portion that constitutes most of this material. The boundaries between the ceramic and polymer portions became more evident with the dissolution of the feldspathic matrix by the acid. Furthermore, microcracks were observed on the surface, revealing that this material seems to have been the most affected by the acid.

On the other hand coffee subgroup with $\mathrm{pH}$ (5.5) recorded highest statistical roughness mean value with PEEK $(0.2439 \pm 0.013)$, The increase in surface 
roughness with coffee subgroup may be due to acidity in coffee which alters surface topography by creating micro- and nano-scale porosities of varying depth and with water might be due to water sorption of the material ${ }^{(33)}$.

\section{Color}

It was found that there was no statistical significant difference between both groups in color change but within the subgroups there was significant change with different $\mathrm{pH}$ media on PEEK and no significant change on Vita Enamic group.

Coffee subgroup recorded statistically significant highest color mean value in both groups, it may be due to smaller molecular size of coffee coupled with water absorption characteristic of the tested materials, creating a stronger staining effect ${ }^{(34,35)}$.

PEEK recorded statistically higher color mean value, this discoloration usually occurs as a result of water sorption by the resin component of the material. The type of resin matrix plays a vital role in the color sustainability of the material.

According to the manufacturer, polymer part in Vita Enamic consists hydrophobic urethane dimethacrylate (UDMA) and hydrophilic triethylene glycol dimethacrylate (TEGDMA). Accordingly, the TEGDMA in Vita Enamic blocks may have resulted in greater water sorption which may have permitted the penetration of any hydrophilic colorant into the resin matrix.

Since UDMA is more hydrophobic than TEGDMA, it is therefore more color stable. Nevertheless, it has been reported that dimethacrylates form cross-linked networks with entrapped unreacted monomers that serve as plasticizers. Such plasticization forms a more open structure, which may facilitate additional water sorption. This may explain how the resin matrix could have contributed to the higher discoloration values obtained by Vita Enamic ${ }^{(36-40)}$.
Previous studies observed that discoloration occurs with Vita Enamic in coffee solution which could be attributed to compatibility of the yellow colorant with the resin matrix. The degree of dye penetration into the resin matrix depends on the dye polarity. Since coffee is a solution of low polarity, its yellow colorant may have facilitated deeper colorant penetration into the resin matrix ${ }^{(31,38,40,41)}$.

\section{Translucency}

Group PEEK showed lower statistical significant value than Vita Enamic in translucency parameter which may be due to the effect of opaque base layer while Vita Enamic is a monolithic material with no base material.

Different solutions showed no significant effect on translucency in both groups, some previous studies confirmed this findings as Eldwakhly et al (42) who evaluated color stability and translucency of different restorative CAD/CAM materials before and after being subjected to different staining solutions(cola, coffe and ginger solutions). Glass ceramic (E-max), high-translucency zirconia (lavaTM Plus), resin nanoceramic (Lava Ultimate), and hybrid ceramic (VITA ENAMIC) CAD/CAM blocks, it was found that staining significantly affected the color $\Delta \mathrm{E}$ of all specimens. No significant differences in $\triangle \mathrm{TP}$ were found between different staining solutions.

Kurt et al ${ }^{(43)}$ evaluated the effects of accelerated artificial aging on the translucency and color stability of monolithic ceramics with different surface treatments. $\Delta \mathrm{E}$ values of the groups exceeded the clinically acceptable level and TP values were not affected by the surface treatment in either material and decreased after aging.

According to the previous results the hypothesis of this study was partially accepted as there was significant change in surface roughness with $\mathrm{pH}$ media on PEEK group and non significant change with Vita Enamic group. Significant change in color stability with PEEK and non siginificant with Vita Enamic. Different pH media did not affect the Translucency in both groups 


\section{CONCLUSION}

Within the limitation of this study, it concluded that:

1. Vita Enamic can maintain smoother surface than PEEK with different $\mathrm{pH}$ media.

2. Vita Enamic can maintain color stability more than PEEK with different $\mathrm{pH}$ media.

3. Vita Enamic has higher translucency than PEEK.

4. Prolonged exposure of polymer materials to weak acids can cause increase in surface roughness, while prolonged exposure to strong acids can decrease surface roughness of the material.

\section{REFERENCES}

1. Vichi A, Louca C, Corciolani G, Ferrari M. Color related to ceramic and zirconia restorations: a review. Dent Mater. 2011;27(1):97-108.

2. Denry I, Kelly JR. Emerging ceramic-based materials for dentistry. Journal of dental research. 2014;93(12):1235-42.

3. Spitznagel FA, Horvath SD, Guess PC, Blatz MB. Resin bond to indirect composite and new ceramic/polymer materials: a review of the literature. J Esthet Restor Dent. 2014;26(6):382-93.

4. Ben-Ur Z, Aviv I, Cardash HS. A modified direct retainer design for distal-extension removable partial dentures. J Prosthet Dent. 1988;60(3):342-4.

5. Gungor MA, Artunc C, Sonugelen M. Parameters affecting retentive force of conus crowns. Journal of oral rehabilitation. 2004;31(3):271-7.

6. Jain C, Bhargava A, Gupta S, Rath R, Nagpal A, Kumar P. Spectrophotometric evaluation of the color changes of different feldspathic porcelains after exposure to commonly consumed beverages. Eur J Dent. 2013;7(2):172-80.

7. Reddy A, Norris DF, Momeni SS, Waldo B, Ruby JD. The $\mathrm{pH}$ of beverages available to the American consumer. Journal of the American Dental Association (1939). 2016;147(4):255.

8. Taufall S, Eichberger M, Schmidlin PR, Stawarczyk B. Fracture load and failure types of different veneered polyetheretherketone fixed dental prostheses. Clin Oral Investig. 2016;20(9):2493-500.
9. Lawson NC, Burgess JO. Gloss and Stain Resistance of Ceramic-Polymer CAD/CAM Restorative Blocks. J Esthet Restor Dent. 2016;28 Suppl 1:S40-5.

10. Abouelatta $\mathrm{OB}$, editor 3D surface roughness measurement using a light sectioning vision system. Proceedings of the world congress on engineering; 2010.

11. Horcas I, Fernandez R, Gomez-Rodriguez JM, Colchero J, Gomez-Herrero J, Baro AM. WSXM: a software for scanning probe microscopy and a tool for nanotechnology. The Review of scientific instruments. 2007;78(1):013705.

12. Barakah HM, Taher NM. Effect of polishing systems on stain susceptibility and surface roughness of nanocomposite resin material. J Prosthet Dent. 2014;112(3):625-31.

13. Kakaboura A, Fragouli M, Rahiotis C, Silikas N. Evaluation of surface characteristics of dental composites using profilometry, scanning electron, atomic force microscopy and gloss-meter. Journal of materials science Materials in medicine. 2007;18(1):155-63.

14. The Glossary of Prosthodontic Terms: Ninth Edition. J Prosthet Dent. 2017;117(5s):e1-e105.

15. Pipko DJ, el-Sadeek M. An in vitro investigation of abrasion and staining of dental resins. Journal of dental research. 1972;51(3):689-705.

16. Hachiya Y, Iwaku M, Hosoda H, Fusayama T. Relation of finish to discoloration of composite resins. Prosthet Dent. 1984;52(6):811-4.

17. Smith DC. Recent developments and prospects in dental polymers. The Journal of Prosthetic Dentistry J. 1962;12(6):1066-78.

18. Kirsch A, Ackermann KL. The IMZ osteointegrated implant system. Dental clinics of North America. 1989;33(4):733-91.

19. Milleding P, Haraldsson C, Karlsson S. Ion leaching from dental ceramics during static in vitro corrosion testing. Journal of biomedical materials research. 2002;61(4): 541-50.

20. Stawarczyk B, Jordan P, Schmidlin PR, Roos M, Eichberger M, Gernet W, et al. PEEK surface treatment effects on tensile bond strength to veneering resins. J Prosthet Dent. 2014;112(5):1278-88

21. Della Bona A, Corazza PH, Zhang Y. Characterization of a polymer-infiltrated ceramic-network material. Dent Mater. 2014;30(5):564-9.

22. Coldea A, Swain MV, Thiel N. Mechanical properties of polymer-infiltrated-ceramic-network materials. Dent Mater. 2013;29(4):419-26. 
23. Belli R, Geinzer E, Muschweck A, Petschelt A, Lohbauer $U$. Mechanical fatigue degradation of ceramics versus resin composites for dental restorations. Dent Mater. 2014;30(4):424-32.

24. Heydecke G, Peters MC. The restoration of endodontically treated, single-rooted teeth with cast or direct posts and cores: a systematic review. J Prosthet Dent. 2002;87(4):380-6.

25. Turgut $\mathrm{S}, \mathrm{Bagis} \mathrm{B}$. Colour stability of laminate veneers: an in vitro study. J Dent. 2011;39 Suppl 3:e57-64.

26. Ren YF, Feng L, Serban D, Malmstrom HS. Effects of common beverage colorants on color stability of dental composite resins: the utility of a thermocycling stain challenge model in vitro. J Dent. 2012;40 Suppl 1:e48-56.

27. Malekipour MR, Sharafi A, Kazemi S, Khazaei S, Shirani F. Comparison of color stability of a composite resin in different color media. Dental research journal. 2012;9(4):4416.

28. Soygun K, Varol O, Ozer A, Bolayir G. Investigations on the effects of mouthrinses on the colour stability and surface roughness of different dental bioceramics. J Adv Prosthodont. 2017;9(3):200-7.

29. Samra AP, Ribeiro DG, Borges CP, Kossatz S. Influence of professional prophylaxis on reducing discoloration of different aesthetic restorative materials. J Dent. 2012;40 Suppl 2:e71-6.

30. Amaya-Pajares SP, Ritter AV, Vera Resendiz C, Henson BR, Culp L, Donovan TE. Effect of Finishing and Polishing on the Surface Roughness of Four Ceramic Materials after Occlusal Adjustment. J Esthet Restor Dent. 2016;28(6):382-96

31. Saba DA, Salama RA, Haridy R. Effect of different beverages on the color stability and microhardness of CAD/ CAM hybrid versus feldspathic ceramic blocks: An invitro study. Future Dental Journal. 2017;3(2):61-6.

32. Cruz MEM, Simoes R, Martins SB, Trindade FZ, Dovigo LN, Fonseca RG. Influence of simulated gastric juice on surface characteristics of CAD-CAM monolithic materials. J Prosthet Dent. 2019.

33. Ramakrishnaiah R, Alkheraif A, Divakar D, Matinlinna J, Vallittu P. The effect of hydrofluoric acid etching duration on the surface micromorphology, roughness, and wettability of dental ceramics. International journal of molecular sciences. 2016;17(6):822.
34. Gujjari ak, M Bhatnagar V, M Basavaraju R. Color stability and flexural strength of poly (methyl methacrylate) and bis-acrylic composite based provisional crown and bridge auto-polymerizing resins exposed to beverages and food dye: An in vitro study. Indian journal of dental research : official publication of Indian Society for Dental Research. 2013;24:172-7.

35. Sham AS, Chu FC, Chai J, Chow TW. Color stability of provisional prosthodontic materials. J Prosthet Dent. 2004;91(5):447-52.

36. Arocha MA, Basilio J, Llopis J, Di Bella E, Roig M, Ardu $\mathrm{S}$, et al. Colour stainability of indirect CAD-CAM processed composites vs. conventionally laboratory processed composites after immersion in staining solutions. J Dent. 2014;42(.7):831-8

37. Awliya WY, Al-Alwani DJ, Gashmer ES, Al-Mandil HB. The effect of commonly used types of coffee on surface microhardness and color stability of resin-based composite restorations. Saudi Dent J. 2010;22(4):177-81.

38. Tuncer D, Karaman E, Firat E. Does the temperature of beverages affect the surface roughness, hardness, and color stability of a composite resin? European journal of dentistry. 2013;7(2):165-71.

39. de Alencar E Silva Leite ML, da Cunha Medeiros E Silva FDS, Meireles SS, Duarte RM, Andrade AKM. The effect of drinks on color stability and surface roughness of nanocomposites. European journal of dentistry. 2014;8(3): 330-6.

40. Acar O, Yilmaz B, Altintas SH, Chandrasekaran I, Johnston WM. Color stainability of CAD/CAM and nanocomposite resin materials. J Prosthet Dent. 2016;115(1):71-5.

41. Borges AL, Costa AK, Saavedra GS, Komori PC, Borges $\mathrm{AB}$, Rode SM. Color stability of composites: effect of immersion media. Acta odontologica latinoamericana : AOL. 2011;24(2):193-9.

42. Eldwakhly E, Ahmed DRM, Soliman M, Abbas MM, Badrawy W. Color and translucency stability of novel restorative CAD/CAM materials. Dental and medical problems. 2019;56(4):349-56.

43. Kurt M, Turhan Bal B. Effects of accelerated artificial aging on the translucency and color stability of monolithic ceramics with different surface treatments. J Prosthet Dent. 2019;121(4):712.e1-.e8. 